\title{
RELAÇÃO ENTRE ESTRUTURA PARA A MELHORIA CONTÍNUA E DESEMPENHO E ESTRUTURA ORGANIZACIONAL
}

\section{RELATIONSHIP BETWEEN ORGANIZATIONAL STRUCTURE FOR CONTINUOUS IMPROVEMENT AND PERFORMANCE}

\author{
Pedro Carlos Oprime \\ Professor Adjunto \\ UFSCar - Universidade Federal de São Carlos \\ DEP - Departamento de Engenharia de Produção \\ São Carlos - São Paulo \\ (16) 3351-9539 \\ pedro@dep.ufscar.br \\ Fabiane Letícia Lizarelli \\ Doutoranda em Engenharia de Produção \\ UFSCar - Universidade Federal de São Carlos \\ DEP - Departamento de Engenharia de Produção \\ São Carlos - São Paulo \\ (16) $9770-8252$ \\ fabianll@uol.com.br
}

\begin{abstract}
RESUMO
O objetivo geral deste artigo é analisar a relação entre a estrutura organizacional (design) para a melhoria contínua e o desempenho das empresas. Tendo por referência um conjunto de variáveis organizacionais, um grupo de empresas amostradas aleatoriamente foi classificado em quatro clusters com diferentes níveis de habilidades para a melhoria contínua. Com base nesse resultado foi possível identificar os comportamentos/habilidades que mais dissimilam os grupos de empresas, bem como a relação entre as habilidades organizacionais e o desempenho das empresas. Outra relação estabelecida foi entre os comportamentos/habilidades organizacionais para a $\mathrm{MC}$ e os sistemas de produção predominante. $\mathrm{O}$ método de pesquisa aplicado foi o estudo de campo através de survey e estudos de caso. Análises estatísticas multivariada indicaram correspondência entre a estrutura organizacional, desempenho e sistemas de produção. Testes de significância estatística mostraram que o grupo de empresas com melhores habilidades para a MC obteve melhores desempenhos, e que empresas com um sistema de produção contínua/ seriada estão mais avanças nas atividades de melhoria contínua que empresas que possuem processos intermitentes.
\end{abstract}

Palavras-chave: Qualidade; Estrutura Organizacional; Melhoria Contínua; Sistema de Produção; Desempenho. 


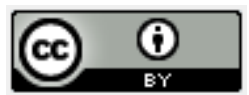

\begin{abstract}
The aim of this article is to examine the relationship between organizational structure (design) for the continuous improvement and performance industrial firms. Based on a set of organizational variables, a group of randomly sampled firms were classified into four clusters with different levels of abilities for continuous improvement. Based on this result was possible to identify the behaviors and skills that most dissimilar groups of companies, the relationship between organizational skills and performance. Other aspect observed was the relationship between the organizational structure for the $\mathrm{MC}$ and the production systems. The research method applied was survey and case studies. The results indicated correlation between the organizational structure, performance and production systems. Tests of statistical significance indicated that the group of companies with the best organizational structure for the $\mathrm{MC}$ has been better performance.
\end{abstract}

Keywords: Quality; Organizational Structure; Continuous Improvement; Production System, Performance.

\title{
1 INTRODUÇÃO
}

A melhoria contínua, MC, é definida como um amplo processo organizacional, focada e sustentada por uma abordagem orientada à melhoria incremental (BESSANT; FRANCIS, 1999). Utilizada como um instrumento gerencial pelas organizações para que melhore sua capacidade competitiva frente às turbulências e incertezas do ambiente externo, a melhoria contínua tem sido implementada por meio de programas formais em muitas empresas, cujo escopo envolve filosofias, conceitos e ferramentas. Muitos desses programas são provenientes de abordagens e sistemas como a do TQM (Total Quality Management), Kaizen, Seis Sigma, Lean Manufacturing, PNQ, ISO 9001 e TPM (Total Productive Maintenance). Como em muitos casos, as empresas implementam várias dessas abordagens paralelamente, isso tende a dificultar a articulação das atividades de melhoria contínua em diferentes níveis da organização.

Um conjunto significativo de fatores associados à melhoria contínua pode ser identificado na literatura, em especial, a necessidade de uma estrutura que suporte e facilite o aprendizado dos indivíduos por meio do compartilhamento das experiências, tanto as positivas quanto as negativas. Outros fatores relacionados ao assunto tratam dentre outros, dos seguintes aspectos: i) análise das estratégias de controle das atividades dos grupos de 


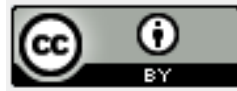

melhoria, podendo estas terem controles rígidos ou uma estrutura mais orgânica (DABHILKAR; BENGTSSON; 2004); ii) análise da importância da cooperação lateral no desenvolvimento das habilidades e competências dos grupos de melhoria (HAMMERSLEY; PINNGTON, 1999; JABNOUN, 2001; LEE, 2004; ABRAHAMSSON; GERDIN, 2006); iii) os níveis de maturidade da MC e o compartilhamento de experiências e aprendizados (BESSANT; FRANCIS, 1999; BESSANT et al., 2000); iv) as ideologias, práticas gerenciais e o processo de renovação organizacional para a melhoria contínua (HARRINGTON, 1995; SAVOLAINEN, 1999); v) a relação entre a melhoria contínua e os sistemas de gestão da qualidade, tal como a ISO 9000 (SACCHETTI, 2007; TERZIOVSKY; POWER, 2007); vi) os mecanismos de suporte e uso de ferramentas para a melhoria contínua (BESSANT et al., 1994; CHAPMAN; HYLAND, 1997; HYLAND et al., 2000; JOINER, 2007); vii) o grau de participação dos indivíduos frente a diferentes modos de organização e suporte às atividades de melhoria (DELBRIDGE; BARTON, 2002); viii) as formas de controle das atividades de MC e suas implicações (DABHILKAR; BENGTSSON, 2004); ix) o uso de indicadores de desempenho e de sistemas de informação para o suporte às atividades de melhoria (BECKET et al., 2000 E BOND, 1999); $\mathrm{x}$ ) os fatores críticos para as atividades de melhoria (ATKINSON, 1994; MARIN-GARCIA et al., 2008); xi) as diferenças e implicações das organizações do tipo top-down e bottom-up para a melhoria contínua (HARRINGTON, 1995; IMAI, 1997; TERZIOVSKI, 2002); e, xii) link entre times e aprendizado organizacional (POWER et al., 2004).

A partir da análise do conjunto de aspectos já abordados pela literatura sobre o assunto, percebe-se que a MC é uma atividade crítica e complexa, o que fica evidente quando se estuda o trabalho de Bessant et al. (2001), o qual apresenta diferentes níveis de maturidade para a melhoria contínua, considerando a evolução nas práticas e habilidades organizacionais. Esses níveis de maturidade são determinados por comportamentos da organização em relação à $\mathrm{MC}$, não só em relação às técnicas e métodos, mas também ao suporte gerencial, à gestão das atividades de MC e ao aprendizado organizacional. E são justamente as habilidades que as organizações adquirem com suas práticas de MC que a conduzem a este aprendizado.

Dada a importância estratégica e a sua complexidade, é importante estudar como a estrutura (design) organizacional para a MC afeta o desempenho das empresas. Tendo por referência as habilidades e comportamentos organizacionais delineados por Bessant et al. 


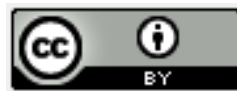

(2001), pretende-se nesta pesquisa: i) classificar as empresas em diferentes grupos com base em sua estrutura organizacional avaliada segundo os seus níveis de comportamentos/habilidades; ii) identificar quais são os comportamentos/habilidades que mais dissimilam os grupos de empresas; iii) analisar as relações entre a estrutura organizacional, e seus níveis de comportamentos/habilidades, com o desempenho das empresas; iv) verificar se há relação entre a estrutura organizacional para a MC e os sistemas de produção predominante nas empresas.

Espera-se com os resultados dessa pesquisa contribuir para a identificação de boas práticas de gestão da MC, além de verificar se há uma situação específica onde essas atividades são desenvolvidas. Como não há um modelo único de estruturar as atividades de MC, em razão das especificidades de cada organização e dá existência de um conjunto de fatores associados a sua implementação, as proposições assumidas neste trabalho são as seguintes:

- Há fatores condicionantes, em especial os associados ao modo de produção/produtos, que facilitam ou restringem as atividades de MC. Desta forma, dependendo do tipo de empresa e seu sistema de produção, existirão diferentes níveis de comportamentos/habilidades da MC.

- Níveis de comportamentos/habilidades organizacionais conduzem as empresas a melhores resultados.

Para validar as proposições desse artigo, foi estudada uma amostra de quarenta e cinco empresas, a maioria com o certificado da ISO 9001, versão 2000 ou algum outro programa, como o TPM e Seis Sigma. As técnicas de pesquisa utilizadas são o survey e estudos de caso. O instrumento de coleta de dados foi baseado nos níveis de comportamento/habilidades para a MC proposta pela teoria, e para análises dos dados foram aplicadas as técnicas de análises estatísticas multivariadas ( $K$-means e análise de correspondência) e teste de independência entre grupos (HARTIGAN et al., 1978). O artigo está estruturado em três seções, além desta introdução e da conclusão. A primeira seção aborda os conceitos e fundamentos da melhoria contínua; a segunda seção detalha o método de pesquisa, e a terceira apresenta os resultados e a análise dos dados levantados. 


\section{REVISÃO DA LITERATURA}

Há vários artigos e textos que abordam diversos aspectos relacionados à melhoria contínua. Primeiramente, há a distinção feita por Imai (1997) entre melhoria contínua e inovação. De modo geral, o autor diferencia as pequenas melhorias, as incrementais, dos grandes saltos de melhoria, sendo que a melhoria foca as atividades de grupos nos locais de trabalho, denominados de Círculos de Controle da Qualidade (CCQ), e a inovação é responsabilidade da administração, cujos projetos são mais complexos e envolvem grandes investimentos. Já o controle da rotina é de responsabilidade do pessoal da linha de produção. Assim, em Imai (1997), é possível identificar diferenças entre inovação, melhoria contínua e controle da rotina.

Há registros de que a melhoria contínua na indústria americana teve início no final do século XIX, mais precisamente entre 1880 e 1894, que corresponde ao período de surgimento, de forma mais estruturada, da chamada administração científica (JHA et al., 1995; BHUIYAN; BAGHEL, 2005).

A idéia de melhoria contínua, como instrumento de gestão, ganhou destaque e operacionalização com a difusão da gestão da qualidade no Japão, que teve como precursores autores americanos como Juran e Deming, na década de 1950. A partir de então a melhoria contínua foi se consolidando, em âmbito mundial, por meio da difusão do modelo japonês de gestão da qualidade (TQC - Total Quality Control).

No Brasil, a partir da década de 1980, aparece o Kaizen, que foi o principal difusor da melhoria contínua no país. Apesar do Kaizen conceituar três tipos de atividades de melhoria contínua (o do individual, o de pequenos grupos e o da administração), o que prevalece no país é a associação da MC às atividades do CCQs (IMAI, 1997).

Mais recentemente, as atividades de melhoria contínua ganharam novos impulsos com as abordagens do Seis Sigma, da Manutenção Produtiva Total (TPM) e da Produção Enxuta. Essas abordagens propõem a eliminação de desperdícios, tais como a redução de estoques, dos tempos de desenvolvimento de novos produtos, redução dos ciclos de produção, redução das áreas ocupadas, redução do tempo de indisponibilidade de equipamentos e melhoria da qualidade de produtos e processos. Cada uma dessas abordagens fornece as bases, teóricas e 


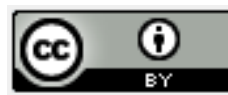

instrumentais, para as empresas organizarem e operacionalizarem as atividades de grupos e indivíduos voltadas para a melhoria de produtos e processos.

Nas duas últimas décadas, vários trabalhos contendo proposições prescritivas ou resultadas de pesquisas de campo têm sido publicados, ampliando o entendimento sobre a melhoria contínua. Caffyn e Bessant (1996); Bessant (1999); Upton (1998); Prajogo e Sohal (2001), Boer e Gertsen (2003) e Tonnessen (2005) são alguns desses autores que introduziram importantes conceitos sobre a MC nessas últimas décadas.

\subsection{Mudanças organizacionais e a organização para a melhoria contínua}

Dentre os fatores relacionados às atividades de melhoria contínua em uma organização, destacam-se: os aspectos culturais e paradigmas vigentes; a organização dos grupos (organizado em força tarefa, em grupos semi-autônomos, com cooperação e comunicação lateral, uso de facilitadores e especialistas em técnicas e métodos de solução de problemas e suporte gerencial); coordenação dos grupos (centralizado ou descentralizado, através de rotinas e métodos especificados e acompanhamento das atividades); domínio e aplicação de técnicas e ferramentas na identificação e solução de problemas (7 ferramentas da qualidade, FMEA, DOE, 7 novas ferramentas da qualidade, dentre outras); desenvolvimento de um sistema de informação para facilitar a coleta e análise de dados de processo e produto, bem como para gerar indicadores de desempenho que facilite e apóie as atividades dos grupos (como o proposto por Becket et al., 2000; Bond, 1999).

Conforme observado, há um conjunto significativo de elementos a ser considerado nas atividades de MC, que não de resume aos métodos, técnicas e ferramentas. Isso foi constatado no início da década de 90 por Atkinson (1994) que apresentou como elementos críticos de um programa de melhoria contínua o comprometimento da gerência, a educação e treinamento dos funcionários, a utilização de medidas de desempenho e o reconhecimento dos grupos/indivíduos. Mais recentemente, Marin-Garcia et al. (2008) reforçaram a importância desses aspectos ao apontarem como fatores críticos para o sucesso da MC o comprometimento e participação dos indivíduos, comprometimentos dos gerentes, liderança do processo, treinamento e a adoção de mecanismos de incentivos. 


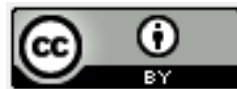

Pode-se afirmar que a melhoria contínua é um processo de renovação organizacional. o que significa introduzir novos comportamentos e reformas na estrutura administrativa, principalmente nas práticas gerenciais (SAVOLAINEN, 1999). O processo de renovação das ideologias e práticas organizacionais ocorre de modo lento, tendo como resultado novos modos de agir incorporado à rotina da organização. Assim, a melhoria contínua tem valores básicos e cabe a organização compreendê-los e articulá-los no seu contexto.

Nessa linha, Savolainen (1999) aponta como um fator crítico de sucesso das atividades de melhoria contínua a introdução de mudanças na ideologia da organização. Segundo esse autor, essa mudança pode ocorrer de três modos distintos: i) conduzida por um líder, representante da alta administração; ii) induzida por um lento processo de mudanças comportamentais, desenvolvendo valores e ideologias que apóiam as atividades de MC, ou; iii) planejada e controlada de modo racional, sustentadas por modelos, tais como os dos sistemas de gestão da qualidade, Seis Sigma ou TPM. Destaca-se nesse escopo a ideologia do TQM (Total Quality Management) que teve um importante papel na difusão dos valores da melhoria contínua consolidada ao longo das últimas décadas pelas idéias introduzidas por vários gurus (GARVIN, 1993; SAVOLAINEN, 1997). Em síntese, a ideologia do TQM é a melhoria contínua de produtos e processos para melhor satisfazer as necessidades dos clientes.

O questionamento que surge é se os três modos isoladamente são suficientes para desenvolver a cultura para a MC. O terceiro modo de se introduzir a MC pode ser o mais comum, porém, a liderança desempenha um papel relevante nesse aspecto, e isso pode ser confirmado quando da análise dos níveis de maturidade baseado nos comportamentos/habilidades, pois é uma forma de avaliar a evolução cultural e das ideologias das práticas de MC nas empresas.

A ideologia é entendida como um conjunto de princípios e idéias próprias de um grupo, é sua convicção íntima, é a base que norteia as decisões e comportamentos na organização (SAVOLAINEN, 1999). Esses princípios e idéias, comungados pela organização, formam um sistema que compreende as crenças, atitudes e valores, que não são aleatórias, mas moldados com o passar do tempo pelas atitudes dos líderes e pelas experiências adquiridas através das boas práticas. Outra evolução da MC é mostrada por Dabhilkar e Bengtsson (2004) que apontam que a transição da MC tradicional, focada nas atividades de grupos constituídos de pessoas de um mesmo local de trabalho que utilizam técnicas e 


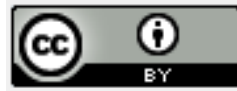

ferramentas na identificação de problemas, para uma organização onde as atividades de melhoria alinham-se aos objetivos estratégicos, cujas metas são monitoradas e passam a ser parte integral da rotina dos indivíduos.

Contribuições nesse sentido vieram dos chamados modelos de excelência e dos sistemas de qualidade, tais como os prêmios nacionais e a ISO 9001 (SACCHETTI, 2007). Terziovsky e Power (2007) identificaram que, quando se adota a ISO 9001, com uma abordagem proativa que suporte as atividades de melhoria contínua, a organização tem um melhor resultado no seu desempenho. O grande benefício dos Sistemas de Gestão da Qualidade é propiciar às organizações uma estrutura básica em termos de padrões e procedimentos que favorecem a introdução das idéias e valores da MC.

Delbridge e Barton (2002) apresentam um amplo espectro de possibilidades de organizar as atividades de melhoria contínua, tais como: i) delegar a especialista a coordenação das atividades, em detrimento, muitas vezes, da responsabilidade de pessoas de chão de fábrica ou mesmo dos gerentes; ii) definir o nível de participação dos indivíduos em projetos de melhoria em função da especialização de indivíduos na solução de problemas (onde há necessidade de maior especialização nos projetos, menor é a porcentagem de participação); iii) definir o nível de centralização das atividades, o que pode estar sob as ordens dos gerentes ou de uma estrutura de decisão mais descentralizada. iv) definir o modo de administrar as atividades da MC, que se dá pelo estabelecimento de rotinas e padrões de procedimentos para os grupos de MC. Em um primeiro momento, os padrões podem ser mais necessários até a organização, grupos e indivíduos adquirem certos comportamentos que conduzam a habilidades organizacionais.

A alta administração, por sua vez, pode adotar alguns mecanismos para garantir que os objetivos estratégicos estejam sendo seguidos e as atividades dos grupos de melhoria, orientadas a esses objetivos. Dentre esses mecanismos, há o BSC (Balanced Scorecard). Porém, esses mecanismos são centralizadores, podendo interferir na proatividade e a autonomia de grupos e indivíduos em propor sugestões de inovações em produtos e processos (DABHILKAR; BENGTSSON, 2004).

O que pode ser um problema dos programas de MC é o formalismo excessivo, que, em certa medida, inibe a participação dos indivíduos ao longo prazo. Por outro lado, a liberdade exagerada pode gerar resultados instáveis pela falta de comprometimento dos indivíduos e da 


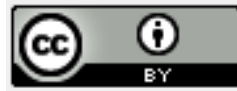

gerência. Recomenda-se, nesses casos, o uso de mecanismos de controle, com amplo envolvimento das pessoas nas atividades de melhoria. É possível, assim, manter um processo de melhoria contínua sustentável, baseado no estímulo a cooperação e comunicação lateral e vertical, e no aprimoramento das habilidades e competência dos indivíduos (ABRAHAMSSON; GERDIN, 2006).

A atitude e a postura gerencial facilitam a comunicação e cooperação lateral, que, juntamente com a autodeterminação dos indivíduos e grupos, estimulam a proatividade em relação à MC. Esses elementos são apresentados por diversos autores (BESSANT; FRANCIS, 1999; SAVOLAINEN, 1999; MURRAY; CHAPMAN, 2003; DABHILKAR; BENGTSSON, 2004; ABRAHAMSSON; GERDIN, 2006).

Segundo Hammersley e Pinngton, (1999), a transformação de CCQ em grupos de melhoria tem como principal característica diferenciadora o controle gerencial e as rotinas que especificam o modo como os grupos devem operar. Nos CCQs não existe controle gerencial rígido e suporte aos projetos, elementos hoje essenciais às atividades de MC.

A MC é incremental. O que distingue a melhoria radical da melhoria incremental, segundo Harrington (1995), é que a melhoria contínua é bottom-up, enquanto a melhoria radical é top-down. Quando se analisa esse aspecto, a literatura indica que os grupos bottomup (debaixo para cima, típicos da abordagem Kaizen) são mais recomendados para a melhoria da produtividade e para a melhoria da satisfação dos clientes. Por outro lado, grupos top-down são mais apropriados para melhoria da competitividade baseada em inovações tecnológicas (TERZIOVSKI, 2002).

Como justificativa da necessidade de formar equipes multidisciplinares, Hyland et al. (2000) dizem que, para identificar e propor soluções para resolver problemas, o conhecimento e habilidades organizacionais podem ser adquiridos de fontes internas e de fontes externas, proveniente de fornecedores, clientes e especialistas. Isso se dá pela cooperação interna ou externa, que implica comunicação clara e direta, confiança entre as partes e, muitas vezes, envolve a solução de conflitos (HARRINGTON, 1995; JABNOUN, 2001; LEE, 2004).

Do exposto, fica claro que a $\mathrm{MC}$ envolve um conjunto de elementos não técnicos, como princípios, valores, crenças, comportamentos etc. O Kaizen, por exemplo, classifica MC como um conceito de melhoria sistemática, permanente e que se realiza em pequenos passos. Outros autores analisam a MC por meio de rotinas e metas definidas para os grupos, que 


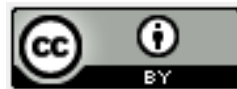

podem, em certa medida, inibir a proatividade dos indivíduos/grupos e a auto-gestão das atividades de melhoria contínua. Essa é uma questão que deve ser avaliada com cuidado, pois também a falta de mecanismos de controle e acompanhamento das atividades pode afetar a eficácia e a efetividade das atividades de melhoria contínua. A MC em projetos não tem a mesma aplicação que em sistemas de produção contínua ou seriada, o que se justifica em parte pela baixa padronização dos processos.

\subsection{Comportamentos e habilidades para a melhoria contínua}

Segundo Bessant et al. (2001), há diferentes níveis de maturidade para a melhoria contínua, considerando-se a evolução nas práticas, princípios e habilidades. Para os autores, são as habilidades que, somadas às práticas e às experiências, conduzem ao aprendizado organizacional e, com isso, à evolução da maturidade da prática de melhoria contínua. Com base nesse raciocínio, é possível estabelecer níveis de maturidade das empresas em relação à melhoria contínua, que são detalhadas no Quadro 1.

\begin{tabular}{|l|l|}
\hline \multicolumn{1}{|c|}{ Nível da Melhoria Contínua } & \multicolumn{1}{|c|}{ Padrões de Comportamento Característicos } \\
\hline $\begin{array}{l}\text { Nível 1 - Pré Melhoria Contínua: } \\
\text { O interesse no conceito já foi iniciado (por } \\
\text { uma crise, ou visita a outra organização, } \\
\text { etc) mas a implementação é feita de forma } \\
\text { ad hoc. }\end{array}$ & $\begin{array}{l}\text { Os problemas são resolvidos aleatoriamente; não existe nenhum } \\
\text { esforço ou estrutura formal para melhorar a organização; } \\
\text { não participação; as soluções visam a benefícios de curto prazo; } \\
\text { não há nenhum impacto estratégico sobre os recursos humanos, } \\
\text { financeiros ou outras metas mensuráveis; gerências e áreas de } \\
\text { apoio não estão conscientes da melhoria contínua ser um } \\
\text { processo. }\end{array}$ \\
\hline $\begin{array}{l}\text { Nível } \\
\text { estruturada: } \\
\text { Há um compromisso formal de construir } \\
\text { um sistema que irá desenvolver a melhoria } \\
\text { contínua por toda a organização. }\end{array}$ & $\begin{array}{l}\text { Mma iniciativa ou organização de melhoria contínua é } \\
\text { introduzida; as áreas de apoio usam processos estruturados de } \\
\text { solução de problemas; uma grande parte das áreas de apoio } \\
\text { participa das atividades de melhoria contínua; as áreas de apoio } \\
\text { são treinadas nas ferramentas básicas de melhoria contínua; há a } \\
\text { introdução de um sistema estruturado de gerenciamento de } \\
\text { idéias; há a introdução de um sistema de reconhecimento e } \\
\text { recompensas; as atividades de melhoria contínua estão integradas } \\
\text { nas operações do dia-a-dia. }\end{array}$ \\
\hline $\begin{array}{l}\text { Nível 3 - Melhoria Contínua orientada } \\
\text { para metas: } \\
\text { Há o compromisso de estabelecer relações } \\
\text { no comportamento da melhoria contínua, }\end{array}$ & $\begin{array}{l}\text { A empresa tem todos os comportamentos do nível 2 mais: } \\
\text { esdobramento formal dos objetivos estratégicos; monitoramento } \\
\text { atividades de melhoria contínua fazem parte das atividades }\end{array}$ \\
\hline
\end{tabular}




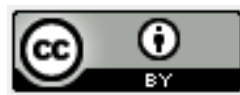

\begin{tabular}{|c|c|}
\hline $\begin{array}{l}\text { relacionando o nível de atuação } \\
\text { operacional com os objetivos estratégicos } \\
\text { mais abrangentes da organização. }\end{array}$ & $\begin{array}{l}\text { principais do negócio; o foco inclui a solução de problemas entre } \\
\text { áreas da empresa (processos inter-organizacionais) ou mesmo } \\
\text { entre empresas (cadeias de produção). }\end{array}$ \\
\hline $\begin{array}{l}\text { Nível } 4 \text { - Melhoria Contínua Proativa: } \\
\text { Há uma tentativa de dar autonomia e poder } \\
\text { aos indivíduos e grupos para gerenciar e } \\
\text { dirigir seus próprios processos e iniciativas } \\
\text { de ações de melhoria. }\end{array}$ & $\begin{array}{l}\text { Todos os comportamentos dos níveis } 2 \text { e } 3 \text { mais: melhoria } \\
\text { contínua desenvolvida e focada na solução proativa de } \\
\text { problemas; altos níveis de práticas de experimentação e } \\
\text { aprendizagem. }\end{array}$ \\
\hline $\begin{array}{l}\text { Nível } 5 \text { - Plena capacidade em Melhoria } \\
\text { Contínua: } \\
\text { Aproxima-se do modelo das organizações } \\
\text { de aprendizagem. }\end{array}$ & $\begin{array}{l}\text { Todos os comportamentos dos níveis } 2,3 \text { e } 4 \text { mais: o } \\
\text { aprendizado organizacional ocorre de modo extensivo e } \\
\text { largamente distribuído; é comum a iniciativa de procura e } \\
\text { solução sistemática de problemas, de forma mais proativa, e a } \\
\text { aquisição e compartilhamento do conhecimento obtido; a } \\
\text { experimentação ocorre de forma abrangente e autônoma, mas } \\
\text { gerenciada. }\end{array}$ \\
\hline
\end{tabular}

Quadro 1: Níveis de maturidade da Melhoria Contínua.

Fonte: BESSANT et al. (2001, p.73)

A melhoria continua envolve um conjunto de comportamentos que são desenvolvidos ao longo do tempo, e desta forma, evoluem. São os comportamentos específicos que determinam as habilidades da organização, e essas são descritas por Bessant e Francis (1999) como sendo as seguintes: 1) Compreendendo a MC: habilidade para articular os valores básicos da $\mathrm{MC}$; 2) Adquirindo o hábito da $\mathrm{MC}$ : habilidade para gerar envolvimento sustentável em MC; 3) Foco na MC: habilidade para unir as atividades de MC aos objetivos estratégicos da organização; 4) Conduzindo a MC: habilidade para conduzir, dirigir e sustentar a criação e a manutenção dos comportamentos para MC; 5) Alinhamento a MC: habilidade para criar consistência entre os valores e os comportamentos para a MC e o contexto da organizacional; 6) Compartilhando a resolução de problemas: habilidades de mover as atividades de MC por meio de barreiras organizacionais; 7) MC no sistema de MC: habilidade para administrar estrategicamente o desenvolvimento da MC; 8) Construindo a organização que aprende: habilidade de aprender por meio da atividade de MC.

Assim, os comportamentos são desenvolvidos e reforçados ao longo do tempo, e podem ser observados de forma direta nas empresas, ou seja, representam a face visível das habilidades. Assim, é possível a avaliação do comportamento das empresas, nas práticas de melhoria, por meio de perguntas que compõem um determinado chek list. Assim, a existência dos comportamentos na empresa pode ser inferida por meio da observação de atitudes, estruturas, procedimentos e práticas. 


\section{MÉTODO DE PESQUISA}

A pesquisa realizada é do tipo descritivo-quantitativa e foi conduzida por meio de um levantamento (survey) e de dois estudos de caso realizados em duas grandes empresas multinacionais localizadas no Estado de São Paulo, que participaram do survey. O objetivo dos estudos de caso é confirmar os resultados obtidos pelo survey. A primeira empresa pertence ao setor automotivo e é uma das empresas pioneiras no Brasil na adoção das normas ISO 9001, Seis Sigma, Lean e de programas de melhoria contínua baseado no Kaizen. A segunda empresa tem plantas em mais de sessenta países e atua em diversos setores da economia, em especial nos setores de mineração e produção de embalagens. A seleção destas duas empresas para o estudo de caso deve-se ao nível de maturidade dos seus programas de qualidade, sendo elas uma referência (benchmarking) das suas matrizes na organização das atividades de melhoria contínua.

Para a coleta de dados (tanto para os estudos de caso como para o survey), o instrumento utilizado foi adaptado do modelo de níveis de maturidade da MC de Bessant e Francis (1999). O quadro 2 relaciona os comportamentos analisados para cada uma das oito habilidades. Já o quadro 3 identifica os efeitos das atividades de MC, em uma escala de 1 a 3 , sendo 3 em larga extensão, 2 em alguma extensão e 1 sem nenhum efeito. Foi aplicada a cada questão do quadro 2 a análise de confiabilidade pelo Alpha de Cronbach e só foram incluídas nas análises as questões que melhoraram a confiabilidade do questionário, ou seja, somente aquelas que aumentaram o valor do Alpha de Cronbach (HAIR et al., 2005).

\begin{tabular}{|l|l|}
\hline Habilidade organizacional & \multicolumn{1}{|c|}{ Comportamentos analisados } \\
\hline $\begin{array}{l}\text { 1. Compreendendo a MC: } \\
\text { habilidade para articular os } \\
\text { valores básicos da MC }\end{array}$ & $\begin{array}{l}\text { A) Pessoas fazem uso de algum método formal para encontrar problemas e } \\
\text { algum ciclo formal para resolvê-los; } \\
\text { B) Quando alguma coisa errada acontece, a reação natural das pessoas de todos } \\
\text { os níveis é procurar as razões do ocorrido ao invés de culpar os indivíduos } \\
\text { envolvidos. }\end{array}$ \\
\hline $\begin{array}{l}\text { 2. Adquirindo o hábito da } \\
\text { MC: habilidade para gerar } \\
\text { envolvimento sustentável em } \\
\text { MC }\end{array}$ & $\begin{array}{l}\text { A) Idéias e sugestões para melhoria são respondidas de forma clara e num tempo } \\
\text { jáminado - ou implementado ou resolvido de outra forma; } \\
\text { B) melhoas (indivíduos/grupos) iniciam e continuam até a finalização atividades }\end{array}$ \\
\hline $\begin{array}{l}\text { 3. Foco na MC: habilidade } \\
\text { para unir as atividades de } \\
\text { MC aos objetivos }\end{array}$ & $\begin{array}{l}\text { A) Pessoas usam técnicas e ferramentas apropriadas para suportar as suas } \\
\text { atividades de melhoria }\end{array}$ \\
\hline
\end{tabular}




\begin{tabular}{|l|l|}
\hline estratégicos da organização & $\begin{array}{l}\text { melhoria e seus impactos na estratégia ou nos objetivos departamentais; } \\
\text { C) Indivíduos e grupos usam a estratégia e objetivos da organização para focar e } \\
\text { priorizar suas atividades de melhoria. }\end{array}$ \\
\hline $\begin{array}{l}\text { 4. Conduzindo a MC: } \\
\text { habilidade para conduzir, } \\
\text { dirigir e sustentar a criação e } \\
\text { a manutenção dos } \\
\text { comportamentos para MC }\end{array}$ & $\begin{array}{l}\text { A) Os gerentes aceitam e quando necessário, agem em relação ao aprendizado } \\
\text { ocorrido; } \\
\text { B) Gerentes de todos os níveis apresentam liderança e comprometimento ativo } \\
\text { em relação à melhoria progressiva; } \\
\text { C) Gerentes conduzem dando o exemplo, se tornando envolvidos ativamente no } \\
\text { planejamento e implementação de melhorias progressivas sistemáticas; } \\
\text { D) Gerentes apóiam a experimentação não punindo erros, mas sim encorajando } \\
\text { através dos mesmos. }\end{array}$ \\
\hline
\end{tabular}

5. Alinhamento a MC: A) Avaliações progressivas garantem que os processos, estruturas e sistemas habilidade para criar organizacionais apóiem consistentemente e reforcem atividades de melhoria;

consistência entre os valores B) Pessoas e times asseguram que seus aprendizados são incorporados à e os comportamentos para a organização fazendo uso dos mecanismos com condição para isso;

MC e o contexto da organizacional

C) Quando uma grande mudança organizacional é planejada, seu impacto potencial no sistema de melhoria organizacional é avaliado e ajustes são feitos quando necessário.

6. Compartilhando a A) Pessoas são orientadas em direção a clientes internos e externos nas suas resolução de problemas: atividades de melhoria;

habilidades de mover as B) Atividades de melhoria relevantes envolvem representantes de diferentes atividades de $\mathrm{MC}$ por meio níveis operacionais;

$\begin{array}{ll}\text { de barreiras organizacionais } & \text { C) Projetos específicos de melhoria ocorrem com clientes e/ou fornecedores }\end{array}$

7. MC no sistema de MC: A) Atividades e resultados de melhorias são continuamente medidos e habilidade para administrar monitorados;

estrategicamente

desenvolvimento da MC

o B) Alta Gerência disponibiliza recursos suficientes (tempo, dinheiro, pessoal) para apoiar o desenvolvimento contínuo do sistema de melhoria da empresa;

C) A organização usa o feedback de clientes e fornecedores como meio de melhorar a performance da companhia.

8. Construindo a organização A) Mecanismos organizacionais apropriados são usados para desenvolver o que que aprende: habilidade de tem sido aprendido através da organização;

aprender por meio da B) Indivíduos procuram por oportunidades de aprendizado/desenvolvimento atividade de MC $\quad$ pessoal (por exemplo, experimentação ativa, relacionados aos objetivos de aprendizado);

C) A organização articula e consolida (captura e compartilha) o aprendizado de indivíduos e grupos.

Quadro 2: As habilidades e comportamentos para a MC.

Fonte: Bessant et al. (2001, p. 72)

Como o Alpha de Cronbach, que mede a correlação global entre as variáveis utilizadas no questionário, sendo essa uma medida da confiabilidade do instrumento de pesquisa, encontrado foi de 0,97 e o valor empírico recomendado é de 0,70, no mínimo (HAIR et al., 2005), pode-se afirmar que o instrumento utilizado era de alta confiabilidade.

Foram enviados e-mails ao gerente da Qualidade de mais de 1.000 empresas do estado de São Paulo. Após 2 meses, 45 empresas haviam feito o preenchimento do questionário, o que representou uma taxa de resposta de aproximadamente $4 \%$. 


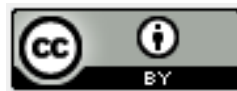

$\mathrm{Na}$ amostra, $82 \%$ das empresas respondentes eram pequenas e médias, de acordo com a classificação do SEBRAE (Serviço Brasileiro de Apoio às Micro e Pequenas Empresas); ou seja, $82 \%$ das empresas da amostra têm entre 20 a 500 funcionários. A outra parte da amostra era constituída por grandes (14\%) e micro empresas $(4 \%)$.

\begin{tabular}{|l|l|}
\hline Rótulo & \multicolumn{1}{c|}{ Tipo de efeito/Resultados } \\
\hline R_199 & Aumento da produtividade \\
\hline R_200 & Melhoria na qualidade de conformação \\
\hline R_201 & Aumento da confiabilidade de entrega \\
\hline R_202 & Redução e lead time \\
\hline R_203 & Redução de custo \\
\hline R_204 & Aumento da satisfação do cliente \\
\hline R_207 & Aumento da habilidade/competência das pessoas \\
\hline R_208 & $\begin{array}{l}\text { Aumento do comprometimento/atitude das pessoas em relação às } \\
\text { mudanças }\end{array}$ \\
\hline R_209 & Melhoria na organização, cooperação e comunicação \\
\hline
\end{tabular}

Quadro 3: Efeito das atividades de MC.

Fonte: Autores

Com relação aos setores da economia, diversos tipos de indústrias estão presentes na amostra, podendo esta ser considerada representativa do amplo espectro de setores que compõem o parque industrial brasileiro. Os setores presentes na amostra são: produtos metálicos (27\%), produtos químicos (18\%), eletrônicos e ópticos (16\%), borrachas e plásticos (11\%), máquinas e equipamentos (11\%), petróleo e equipamentos de transporte (7\%), papel e celulose (4\% das empresas), fabricação de outros produtos minerais não metálicos $(2 \%)$, e outros setores $(4 \%)$.

As análises estatísticas aplicadas foram os testes de independência de grupos e variáveis e a análise de K-means. Utilizou-se o Software Statistica v. 9.0.

\section{APRESENTAÇÃO E ANÁLISE DOS DADOS}

Foram solicitados aos respondentes que informassem em que grau as suas empresas tinham as habilidades/comportamentos organizacionais descritos no quadro 2. Foi adotada uma escala de 1 a 5 (1-discordo completamente a 5-concordo plenamente), para avaliar o grau de concordância com a afirmativa sobre cada um dos comportamentos. 


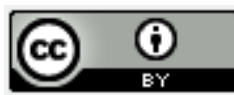

Quatro grupos (cluster) de empresas foram formados com diferentes níveis de comportamentos/habilidades (figura 1). As empresas do grupo/cluster 1 têm menor nível de comportamento/habilidade, já as empresas do grupo/cluster 4 têm maior nível, segundo as percepções dos respondentes. Os clusters/grupos de empresas 2 e 3 podem ser classificados em intermediário inferior e intermediário superior, respectivamente. No cluster 1 foram agrupadas $11 \%$ das empresas da amostra, no cluster 2 foram agrupadas 29\%, no cluster 3 foram agrupadas $31 \%$ e no cluster 4 foram agrupadas $29 \%$ das empresas.

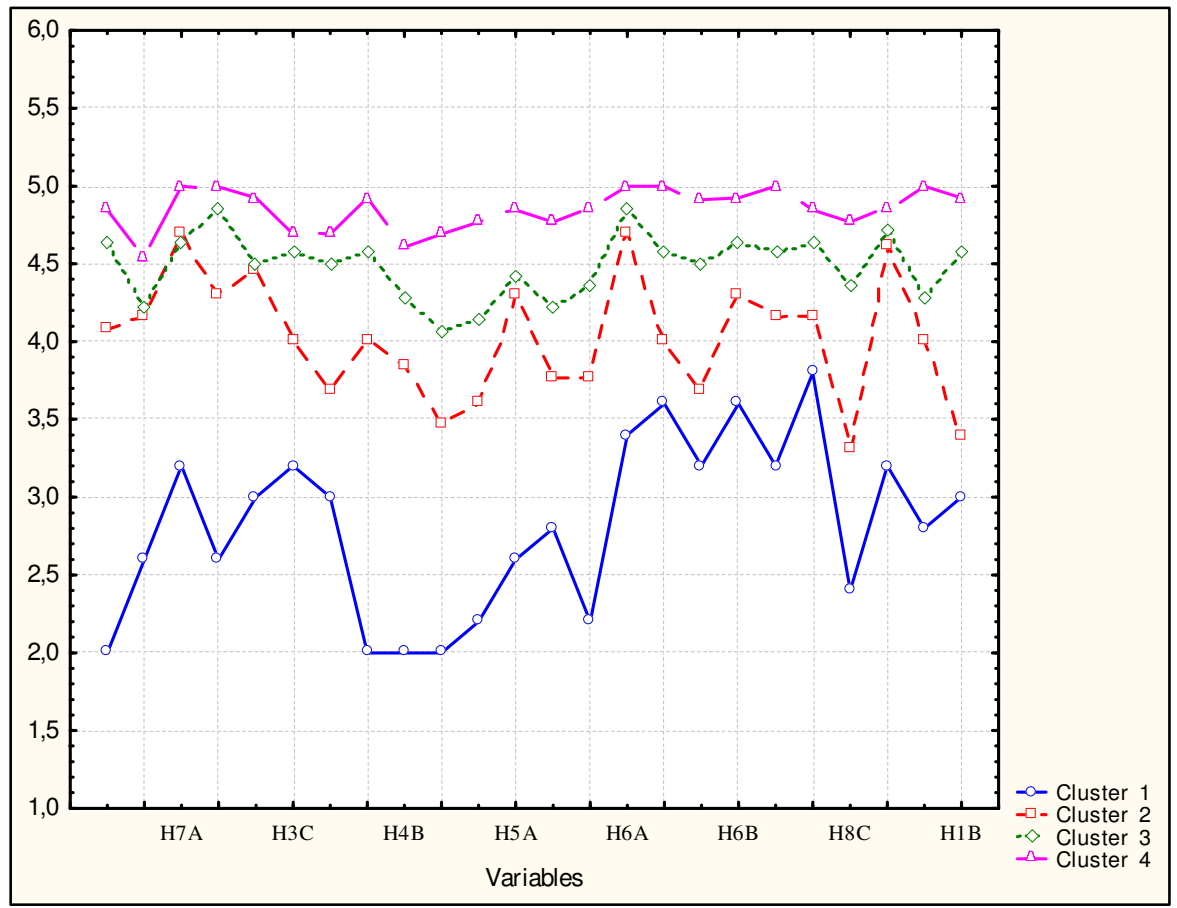

Figura 1: Cluster das empresas obtido a partir das análises estatísticas. Fonte: STATISTICA, v. 9.0

Há comportamentos que produzem maior dissimilaridade entre os clusters, como mostram o gráfico 1 e a tabela 1 . A habilidade identificada como 4 no quadro 2 , com seus respectivos comportamentos, é a que mais produziu dissimilaridade entre as empresas do grupo/cluster 4 em relação às empresas dos grupos/clusters 1 e 2, conforme indica o nível de significância da tabela 1 . O que se constata a partir desses resultados é que a habilidade para conduzir, dirigir e sustentar a criação e a manutenção dos comportamentos para MC é a que mais diferencia os grupos de empresas e é a que teve menor média geral (Tabela 1). 
Tabela 1: Teste de significância dos comportamentos para a MC

\begin{tabular}{|c|c|c|c|c|c|c|c|}
\hline \multirow{2}{*}{ Comportamentos } & \multicolumn{6}{|c|}{ Nível de Significância, $p$} & \multirow{2}{*}{$\begin{array}{l}\text { Média } \\
\text { Geral }\end{array}$} \\
\hline & $(4-1)$ & $(4-2)$ & $(4-3)$ & $(3-1)$ & $(3-2)$ & $(2-1)$ & \\
\hline $\mathrm{H} 4 \mathrm{C}$ & 0,000 & 0,000 & 0,001 & 0,000 & 0,112 & 0,001 & 3,56 \\
\hline H4D & 0,000 & 0,000 & 0,004 & 0,000 & 0,077 & 0,004 & 3,68 \\
\hline H4B & 0,000 & 0,003 & 0,183 & 0,000 & 0,083 & 0,000 & 3,69 \\
\hline $\mathrm{H} 8 \mathrm{C}$ & 0,000 & 0,000 & 0,049 & 0,000 & 0,001 & 0,031 & 3,71 \\
\hline $\mathrm{H} 5 \mathrm{~B}$ & 0,000 & 0,000 & 0,014 & 0,000 & 0,018 & 0,000 & 3,79 \\
\hline $\mathrm{H} 4 \mathrm{~A}$ & 0,000 & 0,000 & 0,027 & 0,000 & 0,022 & 0,000 & 3,87 \\
\hline $\mathrm{H} 2 \mathrm{~A}$ & 0,000 & 0,133 & 0,174 & 0,000 & 0,690 & 0,001 & 3,88 \\
\hline $\mathrm{H} 2 \mathrm{~B}$ & 0,000 & 0,000 & 0,005 & 0,000 & 0,013 & 0,003 & 3,89 \\
\hline H8A & 0,000 & 0,003 & 0,279 & 0,000 & 0,074 & 0,000 & 3,89 \\
\hline H1B & 0,000 & 0,000 & 0,067 & 0,001 & 0,000 & 0,428 & 3,97 \\
\hline H8B & 0,000 & 0,000 & 0,440 & 0,000 & 0,000 & 0,045 & 3,97 \\
\hline $\mathrm{H} 5 \mathrm{C}$ & 0,000 & 0,000 & 0,000 & 0,001 & 0,189 & 0,010 & 4,02 \\
\hline $\mathrm{H} 5 \mathrm{~A}$ & 0,000 & 0,023 & 0,014 & 0,000 & 0,902 & 0,000 & 4,05 \\
\hline $\mathrm{H} 2 \mathrm{C}$ & 0,000 & 0,000 & 0,029 & 0,008 & 0,036 & 0,250 & 4,08 \\
\hline $\mathrm{H} 3 \mathrm{C}$ & 0,000 & 0,002 & 0,695 & 0,000 & 0,006 & 0,027 & 4,12 \\
\hline $\mathrm{H} 3 \mathrm{~A}$ & 0,000 & 0,000 & 0,153 & 0,000 & 0,008 & 0,000 & 4,19 \\
\hline H3B & 0,000 & 0,005 & 0,027 & 0,000 & 0,585 & 0,001 & 4,22 \\
\hline H7B & 0,000 & 0,000 & 0,004 & 0,005 & 0,087 & 0,017 & 4,23 \\
\hline $\mathrm{H} 1 \mathrm{~A}$ & 0,003 & 0,003 & 0,019 & 0,079 & 0,167 & 0,439 & 4,29 \\
\hline $\mathrm{H} 7 \mathrm{C}$ & 0,000 & 0,325 & 0,457 & 0,001 & 0,841 & 0,001 & 4,34 \\
\hline H6C & 0,002 & 0,000 & 0,199 & 0,021 & 0,035 & 0,167 & 4,36 \\
\hline H6B & 0,001 & 0,016 & 0,067 & 0,016 & 0,306 & 0,107 & 4,37 \\
\hline H7A & 0,000 & 0,038 & 0,011 & 0,001 & 0,603 & 0,000 & 4,38 \\
\hline H6A & 0,000 & 0,038 & 0,153 & 0,001 & 0,430 & 0,002 & 4,49 \\
\hline
\end{tabular}

Fonte: STATISTICA, v. 9.0

A habilidade de mover as atividades de MC por meio de barreiras organizacionais (habilidade 6 do quadro 2) é a que teve maior média. Há evidência estatística de que os comportamentos e sua respectiva habilidade relacionados à condução da MC (habilidade 4) são menores que à habilidade de compartilhar a resolução de problemas (habilidade 6), o que pode ser observado na tabela 2 .

Poder-se-ia dizer, com base nos resultados da amostra, que há um grupo significativo de empresas da população pesquisada que está no nível 4 de maturidade (Quadro 1) em várias das habilidades apresentadas no quadro 2, como pode ser observado no figura no cluster 4. Entretanto, a habilidade para conduzir o processo de melhoria contínua, cujos comportamentos estão centrados nas gerências, é uma das habilidades mais frágeis dentre todos os clusters. Na mesma figura 1 é possível identificar a existência de grupos de empresas 


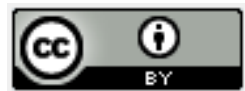

cujos comportamentos/habilidades pode colocá-las no nível 2, caminhando para o nível 3 de maturidade.

Tabela 2: Teste de independência entre os comportamentos das habilidades 4 e 6

\begin{tabular}{ccccccc}
\hline & & $\begin{array}{c}\text { Média da } \\
\text { habilidade 4 }\end{array}$ & $\begin{array}{c}\text { Média da } \\
\text { habilidade 6 }\end{array}$ & t-value & $\begin{array}{c}\text { Graus de } \\
\text { Liberdade }\end{array}$ & p \\
\hline H4B vs. & H6A & 4,000 & 4,689 & $-3,969$ & 88 & 0,00015 \\
\hline H4B vs. & H6B & 4,000 & 4,511 & $-2,860$ & 88 & 0,00529 \\
\hline H4B vs. & H6C & 4,000 & 4,467 & $-2,795$ & 88 & 0,00637 \\
\hline H4C vs. & H6A & 3,844 & 4,689 & $-4,789$ & 88 & 0,00001 \\
\hline H4C vs. & H6B & 3,844 & 4,511 & $-3,675$ & 88 & 0,00041 \\
\hline H4C vs. & H6C & 3,844 & 4,467 & $-3,664$ & 88 & 0,00042 \\
\hline H4D vs. & H6A & 3,956 & 4,689 & $-4,093$ & 88 & 0,00009 \\
\hline H4D vs. & H6B & 3,956 & 4,511 & $-3,017$ & 88 & 0,00334 \\
\hline H4D vs. & H6C & 3,956 & 4,467 & $-2,958$ & 88 & 0,00397 \\
\hline
\end{tabular}

Fonte: STATISTICA, v. 9.0

A Tabela 3 mostra o grau de percepção de ganhos (impacto nos resultados do Quadro 3) pelas empresas do cluster 4 e do cluster 1. Aplicando o teste de independência entre grupos, na tabela 3, verifica-se que há dependência entre os clusters formados (clusters 4, 3, 2 e 1) e os resultados. Assim, há evidência estatística de que as empresas que possuem uma estrutura organizacional (design) mais adequado (caso das empresas do cluster 4) em relação a outras empresas menos estruturadas (caso das empresas do cluster 1), avaliadas estas com base nos comportamentos/habilidades do Quadro 2, obtêm maior confiabilidade de entrega (variável R_201), aumento na satisfação dos clientes (variável R_204), aumento da habilidade/competência das pessoas (R_207), aumento do comprometimento (R_2008) e melhoria na organização e cooperação (R_2009).

Tabela 3: Relação entre os resultados e os clusters 4 e 1

\begin{tabular}{c|c|c|c|c|c}
\hline $\begin{array}{c}\text { Variáveis } \\
\text { Dependentes }\end{array}$ & $\begin{array}{c}\text { Media } \\
\text { Cluster 4 }\end{array}$ & $\begin{array}{c}\text { Média } \\
\text { Cluster 1 }\end{array}$ & t-valor & Gl & p \\
\hline R_199 & 2,462 & 2,200 & 0,990 & 16 & 0,337 \\
\hline R_200 & 2,923 & 2,600 & 1,685 & 16 & 0,111 \\
\hline R_201 & 2,923 & 2,200 & 4,187 & 16 & 0,001 \\
\hline R_202 & 2,308 & 2,000 & 1,405 & 16 & 0,179 \\
\hline R_203 & 2,385 & 2,000 & 1,099 & 16 & 0,288 \\
\hline R_204 & 2,846 & 2,000 & 2,696 & 16 & 0,016 \\
\hline R_207 & 2,692 & 1,600 & 3,398 & 16 & 0,004 \\
\hline R_208 & 2,538 & 1,600 & 3,389 & 16 & 0,004 \\
\hline R_209 & 2,769 & 2,000 & 3,849 & 16 & 0,001 \\
\hline
\end{tabular}

Fonte: STATISTICA, v. 9.0 


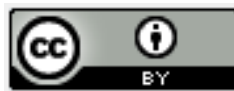

A figura 2 mostra a correspondência entre os níveis de comportamento/habilidades identificados através dos clusters e o tipo de sistema de produção. Verifica-se que há relação entre os níveis de comportamento/habilidades e o sistema de produção predominante da empresa. As empresas do cluster 4 (as mais evoluídas nas habilidades organizacionais) estão relacionadas ao sistema de produção contínua, as do cluster 3 ao sistema de produção em linha, as do cluster 2 à produção em lote/job shop e as do cluster 1 a projetos. Isso indica que as empresas com menos maturidade nas atividades de MC são as que trabalham por projetos (produção intermitente). Esse resultado está de acordo com a percepção dos pesquisadores e também está de acordo com o encontrado na literatura.

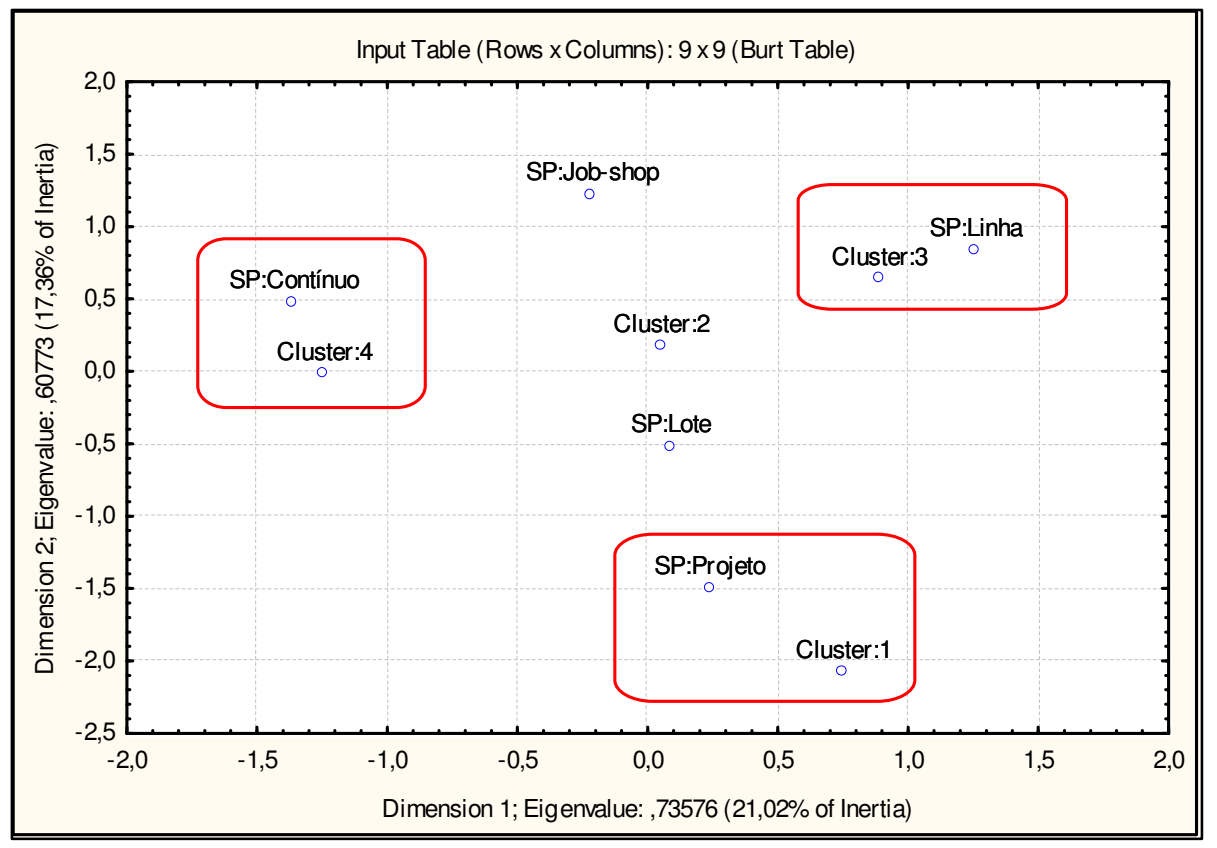

Figura 2: Correspondência entre os níveis de comportamento e o sistema de produção.

Fonte: STATISTICA, v. 9.0.

\subsection{Estudos de Caso}

Os resultados dos dois estudos de caso, que são mostrados no Quadro 4, indicam que as empresas não estão no nível pleno de maturidade das atividades de MC. Indicam também que as habilidades para conduzir, dirigir e sustentar as atividades de melhoria contínua são as mais frágeis. Entretanto, percebe-se a adoção de sistemas, no caso da empresa $A$, que 


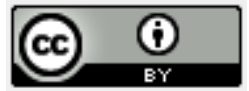

compartilha o uso de boas práticas é um avanço significativo na direção de empresas que aprendem, onde o aprendizado organizacional ocorre de modo extensivo e largamente distribuído.

\begin{tabular}{|c|c|c|}
\hline $\begin{array}{c}\text { Habilidade } \\
\text { organizacional }\end{array}$ & Empresa A & Empresa B \\
\hline $\begin{array}{l}\text { 1. Compreendendo a } \\
\text { MC: habilidade para } \\
\text { articular os valores } \\
\text { básicos da MC }\end{array}$ & $\begin{array}{l}\text { Em geral as pessoas fazem uso de } \\
\text { algum método formal para encontrar } \\
\text { problemas. Na maioria das vezes, } \\
\text { procuram-se as razões do ocorrido ao } \\
\text { invés de culpar os indivíduos } \\
\text { envolvidos. }\end{array}$ & $\begin{array}{l}\text { Somente em casos as pessoas fazem uso de } \\
\text { algum método formal para encontrar } \\
\text { problemas. A reação natural das pessoas diante } \\
\text { de um problema é procurar as razões do } \\
\text { ocorrido ao invés de culpar os indivíduos } \\
\text { envolvidos. }\end{array}$ \\
\hline $\begin{array}{l}\text { 2. Adquirindo o hábito } \\
\text { da MC: habilidade para } \\
\text { gerar envolvimento } \\
\text { sustentável em MC }\end{array}$ & $\begin{array}{l}\text { Idéias e sugestões para melhoria são } \\
\text { respondidas de forma clara e num } \\
\text { tempo já determinado }- \text { ou } \\
\text { implementado ou resolvido de outra } \\
\text { forma; } \\
\text { Pessoas (indivíduos/grupos) iniciam e } \\
\text { continuam até a finalização atividades } \\
\text { de melhoria; eles participam do } \\
\text { processo; } \\
\text { Pessoas usam técnicas e ferramentas } \\
\text { apropriadas para suportar as suas } \\
\text { atividades de melhoria }\end{array}$ & $\begin{array}{l}\text { Idéias e sugestões para melhoria nem sempre } \\
\text { são respondidas ou implementadas. } \\
\text { Nem sempre as pessoas/grupos iniciam e } \\
\text { continuam até a finalização atividades de } \\
\text { melhoria; } \\
\begin{array}{l}\text { Pessoas usam técnicas e ferramentas } \\
\text { apropriadas para suportar as suas atividades de } \\
\text { melhoria }\end{array}\end{array}$ \\
\hline $\begin{array}{l}\text { 3. Foco na MC: } \\
\text { habilidade para unir as } \\
\text { atividades de MC aos } \\
\text { objetivos estratégicos } \\
\text { da organização }\end{array}$ & $\begin{array}{l}\text { Melhoria é uma parte integral do } \\
\text { trabalho dos indivíduos ou dos grupos, } \\
\text { não é uma atividade paralela. } \\
\text { Indivíduos e grupos monitoram/medem } \\
\text { os resultados de suas atividades de } \\
\text { melhoria e seus impactos na estratégia } \\
\text { ou nos objetivos departamentais. } \\
\text { Indivíduos e grupos usam a estratégia e } \\
\text { objetivos da organização para focar e } \\
\text { priorizar suas atividades de melhoria. }\end{array}$ & $\begin{array}{l}\text { Melhoria não é ainda uma parte integral do } \\
\text { trabalho dos indivíduos ou dos grupos, é uma } \\
\text { atividade paralela; }\end{array}$ \\
\hline $\begin{array}{lr}\text { 4. Conduzindo a } & \text { MC: } \\
\text { habilidaderrar } & \text { para } \\
\text { conduzir, dirigir } & \text { e } \\
\text { sustentar a criação e } & \text { a } \\
\text { manutenção r } & \text { dos } \\
\text { comportamentos } & \text { para } \\
\text { MC } & \end{array}$ & $\begin{array}{l}\text { Há reclamações quanto a postura dos } \\
\text { gerentes quanto à } \mathrm{MC} \text {, falta pro } \\
\text { atividade e liderança gerencial. }\end{array}$ & $\begin{array}{l}\text { Os gerentes aceitam e quando necessário, } \\
\text { agem em relação ao aprendizado ocorrido. } \\
\text { Entretanto há descontinuidade dos projetos de } \\
\text { melhoria contínua. Falta liderança gerencial } \\
\text { para a MC. O aspecto positivo é que os } \\
\text { gerentes apóiam a experimentação. }\end{array}$ \\
\hline $\begin{array}{l}\text { 5. Alinhamento a } \mathrm{MC} \text { : } \\
\text { habilidade para criar } \\
\text { consistência entre } \text { os } \\
\text { valores } \quad \text { e } \\
\text { comportamentos para a } \\
\text { MC e o contexto da } \\
\text { organizacional }\end{array}$ & 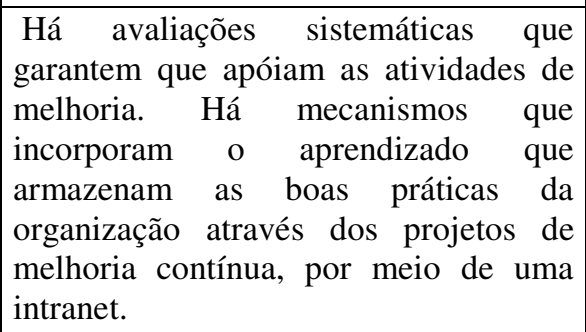 & $\begin{array}{l}\text { Em geral, as experiências boas são } \\
\text { armazenadas em forma de procedimentos e } \\
\text { rotinas de trabalho. Entretanto, não há um } \\
\text { sistema } \quad \text { que armazene } \\
\text { experiências/resultados de projetos } \\
\text { melhoria. }\end{array}$ \\
\hline
\end{tabular}




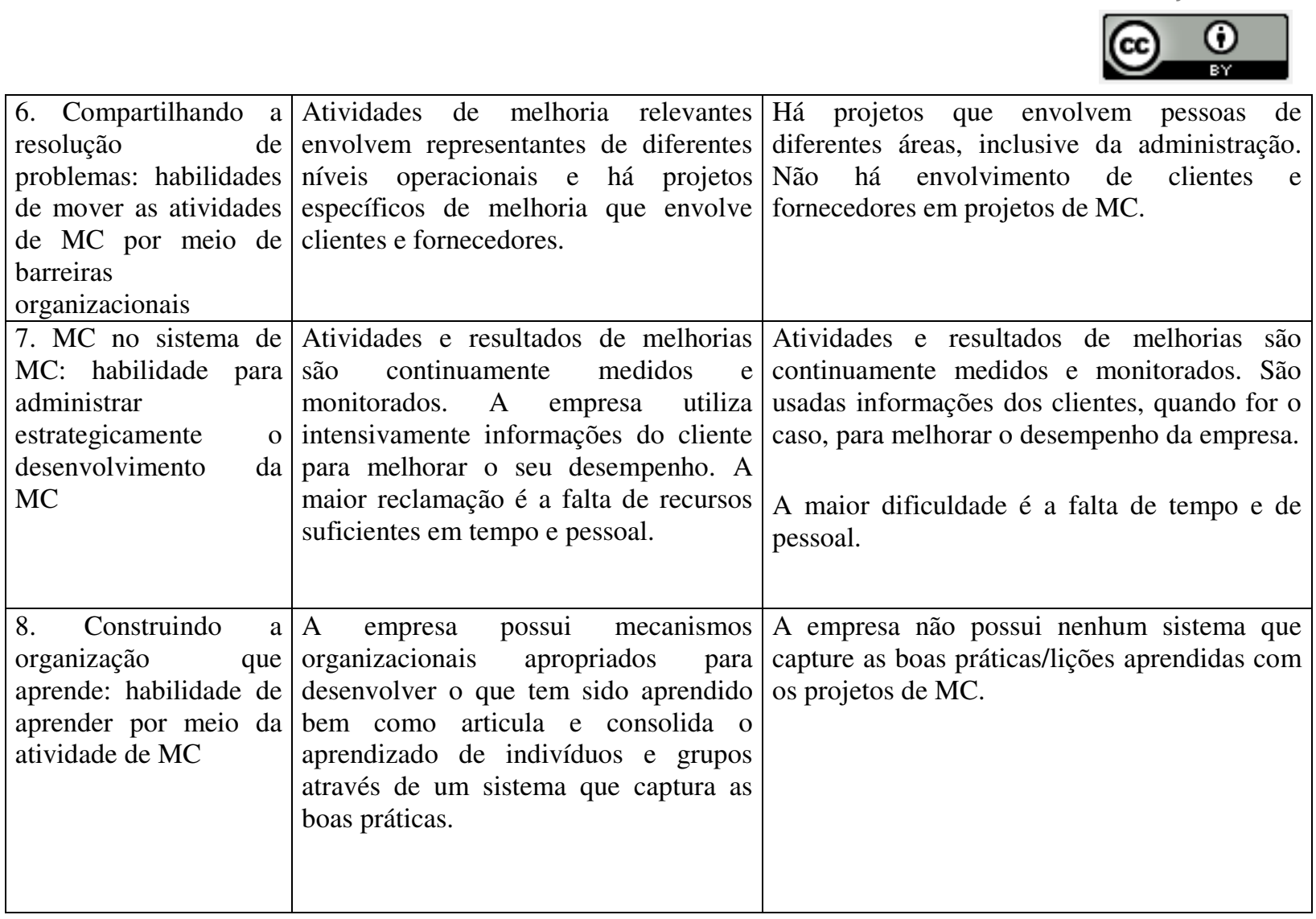

Quadro 4: Comparação das análises entre as duas empresas estudadas

Fonte: Autores

\section{CONCLUSÕES}

Com base nos comportamentos e habilidades organizacionais, foi possível classificar as empresas em grupos de acordo com o design das suas estruturas organizacionais e seus processos de implementação da melhoria contínua. De acordo com essa classificação, testes de significância estatística indicaram que o grupo de empresas com estrutura organizacional com melhores habilidades para a MC obteve melhores desempenho.

Foram identificados quatro grupos de empresa, sendo que um dos grupos apresentou níveis de habilidades organizacionais bem superiores às demais empresas, os que as enquadram em um elevado nível de maturidade organizacional para a MC. Já outro grupo, cerca de $13 \%$ da amostra, teve baixos níveis nos comportamentos avaliados. Todos os comportamentos produziram dissimilaridades entre os grupos formados, entretanto, destacouse o baixo desempenho dentre as empresas na habilidade para conduzir, dirigir e sustentar a MC. Os comportamentos relacionados a essa habilidade estão centrados nos gerentes. Esse resultado mostra a importância dos gerentes como líderes e sustentadores da MC nas 


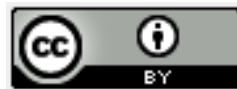

organizações, o que exige em muitos casos a introdução de novos comportamentos e reformas na estrutura administrativa. Para alguns autores, o TQM é um dos mecanismos que pode contribuir para o desenvolvimento de comportamentos que propiciam a organização novas habilidades para a MC.

Os principais aspectos constatados nos estudos de casos foram: o uso de especialistas no apoio aos projetos, o nível de participação dos indivíduos de acordo com a especialização. Há uma tendência das empresas darem aos indivíduos e grupos maior liberdade, baseado no estímulo à cooperação, o empreendedorismo e a criatividade.

Uma das habilidades mais fortes identificadas no survey é o compartilhamento de solução de problemas. A necessidade de formar equipes multidisciplinares para identificar e propor soluções para resolver problemas, demanda conhecimentos e habilidades organizacionais que podem ser adquiridas de fontes internas e externas, proveniente de fornecedores, clientes e especialistas. Isso se dá pela cooperação interna ou externa, que implica comunicação clara e direta e confiança entre as partes.

Verificou-se também que há uma relação entre os níveis de comportamentos/habilidades das empresas e o sistema de produção predominante. Empresas com um sistema de produção contínua ou seriada têm mais habilidades para a MC que empresas que trabalham por projetos. Portanto, o tipo de sistema de produção favorece as atividades de MC. Desta forma, verifica-se que há dependência estatística entre o tipo de produção e o nível de maturidade dos comportamentos/habilidades para a MC.

A principal implicação deste trabalho é a orientação aos gerentes e coordenadores de projetos/programas de melhoria contínua é a necessidade de um enfoque estratégico, de longo prazo, sustentável e suportado pela alta administração da empresa. Sem isso, o desenvolvimento da cultura da MC, de seus comportamentos e habilidades torna-se tarefa complexa, gerando descrença e desconfiança. Nos estudos de caso, a principal dificuldade e a descontinuidade dos programas e a falta de suporte gerencial.

As proposições formuladas nos objetivos desse trabalho foram confirmadas. Apesar do desenvolvimento teórico no assunto, há ainda muitas dúvidas de como organizar e implementar os programas de MC. Adoção de abordagens como a do TPM e Seis Sigma facilita a implementação, entretanto há dificuldade de contabilizar financeiramente os ganhos desses programas. Uma das dificuldades dos coordenadores é quantificar ganhos para 


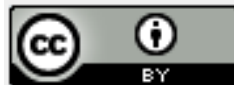

medidas de desempenho estratégico, como redução do leed time de fabricação. Outro desempenho de ser quantifico por esses programas é a motivação dos grupos e indivíduos que participam desses programas.

\section{REFERÊNCIAS}

ABRAHAMSSON, G; GERDIN, J. Exploiting institutional contradictions: the role o management accounting in continuous improvement implementation, Qualitative Research in Accounting \& Management, v. 3, n. 2, p. 126-144, 2006.

ATKINSON, C. Continuous improvement: the Ingredients of change. International Journal of Contemporary Hospitality Management, v. 6, n.1/2, p. 6-8, 1994.

BECKET, A.J.; WAINWRIGHT, C.E.R.; BANCE, D. Implementing an industrial continuous improvement systems: a knowledge management case study. Industrial Management \& Data Systems. v.100, n.7, p.330-338, 2000.

BESSANT, J; CAFFYN, S.; GILBERT, J.; Harding, R.; Webb, S. Rediscovering continuous improvement. Technovation, v. 14, n. 1, p. 17-29, 1994.

BESSANT, J.; CAFFYN, S. High involvement innovation. International Journal of Techology and Management. v.14, n.1, p. 7-28, 1997.

BESSANT, J. Developing continuous improvement capability. International Journal of Innovation Management, v. 8, n.3, p.409-429, 1999.

BESSANT, J.; CAFFYN, S.; GALLAGHER, M. An evolutionary model of continuous improvement behavior, Technovation, v.21, n.2, p. 67-77, 2001.

BESSANT, J.; FRANCIS, D. Developing strategic continuous improvement capability. International Journal of Operation \& production Management, v. 19, n. 11, p.1106-1119, 1999.

BHUIYAN, N.; BAGHEL, A. An overview of continuous improvement: from the past to the present. Management Decision, v. 43, n. 5, p. 761-771, 2005.

BOER, H.; GERTSEN F. From continuous improvement to continuous innovation: a (retro)(per)spective. International Journal Technology Management, v. 26, n. 8, p. 805$827,2003$.

BOND, T.C. The role of performance measurement in continuous improvement. International Journal Operation \& Production Management, v. 19, n. 12, p. 1318-1334, 1999. 


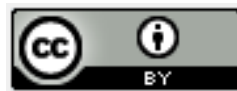

CAFFYN, S.; BESSANT, J. A capability-based model for continuous improvement, INTERNATIONAL CONFERENCE OF THE EUROMA, 3. 1996. Proceedings. London, 1996.

CHAPMAN, R.L.; HYLAND, P (1997). Continuous improvement strategies across selected australian canufacturing sectors, Benchmarking for Quality Management \& Technology, v. $4, \mathrm{n} 1, \mathrm{p} .175-188,1997$.

DABHILKAR, M; BENGTSSON, L. Balanced scorcards for strategic and sustainnable continuous improvement capability. Journal of Manufacturing Techonology Management, v. 15 , n. 4 , p.350-359, 2004.

DELBRIDGE, R; BARTON, H. Organizing for continuous improvement: structures and roles in auto components plants. International Journal of Operations and Production Management, v. 22, n. 6, p. 680-692, 2002.

GARVIN, D. Building a learning organization. Harvard Business Review, p. 78-91, July/Aug., 1993.

HAIR Jr., J. F. et al. Análise multivariada de dados. 5. ed. Porto Alegre: Bookman, 2005. $593 \mathrm{p}$.

HARTIGAN, J.A.;WONG, M.A. A k-means clustering algorithm. Appllied Statistics, v.28, n.100, 1978.

HAMMERSLEY, G.; PINNGTON, A. Employee response to continuous improvement groups. The TQM Magazine, v. 11, n. 1, p.29-34, 1999.

HARRINGTON, H.J. Continuous versus breakthrough improvement: finding the right answer. Business Process Re-engineering \& Management Journal, v. 1, n 3, p. 31-45, 1995.

HYLAND, P. et al. A comparison of Australian firms and their use of continuous improvement tools. The TQM Magazine, v. 12, n. 2, p. 117-124, 2000.

IMAI, M. Gemba Kaisen: a common sense, low-cost approach to management. New York: McGraw-Hill, 1997.

JABNOUN, N. Value underlying continuous improvement. The TQM Magazine, v. 13, n 6, p. 381-387, 2001.

JHA, S.; NOORI, H.; MICHELA, J. L. The dynamics of continuous improvement. International Journal of Quality Science, v. 1, n. 1, p. 19-47, 1995.

JOINER, T.A. Total quality management and performance: the role of organization support and co-worker support. International Journal of Quality \& Reliability Management, v. 24, n. 6, p.617-627, 2007. 


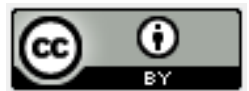

LEE, H.J. The Role Competence-based trust and organizational identification in continuous improvement. Journal of Managerial Psychology, v. 19, n. 6, p. 623-639, 2004.

MARIN-GARCIA, J A.; PARDO-DEL-VAL, M.; BONAVIA, T. Análisis de programas de mejora continua: un estudio longitudinal en una empresa industrial. Gestão e Produção, v.15, n. 3, p.433-447, 2008.

MUDRAK, T.; ANDREAS, W.; WUBBEN, E. Innovation process and innovativeness of facility management organizations. Facilities, v .23, n. 34, p. 103-118, 2005.

MURRAY, P.; CHAPMAN, R. From continuous improvement to organizational learning: development theory. The Learning Organization, v. 10, n. 5, p. 272-282, 2003.

POWER, J.; WADDELL, D. The link between self-managed work teams and learning organizations using performance indicators. The Learning Organization, v. 11, n. 3, p. 244259, 2004.

PRAJOGO, D. I.; SOHAL, A. S. TQM and innovation: a literature review and research framework. Technovation, n.21, n.10, p. 532-558, 2001.

SACCHETTI, L. ISO Quality as a driver of Continuous Improvement. Perfomance Measurement and Metrics, vol. 8, n. 2, p. 88-97. 2007.

SAVOLAINEN, T. Development of Quality-oriented Management Ideology. n.37, 1997.

SAVOLAINEN, T.I. Cycles of Continuous Improvement-Ralizing Competitive Advantage Trough Quality. Internation Journal of Operations \& Production Management, v. 19, n. 11, p.1203-1222, 1999.

TERZIOVSKY, M. Achieving Performance Through an Integrated Strategy of Radical Innovation and Continuous Improvement. Business Excellence, v. 6, n. 2, p. 5-24, 2002.

TERZIOVSKY, M.; POWER, D. Increasing ISO9000 certification benefits: a continuous improvement approach. International Journal Quality \& Reliability Management, v. 24, n. 2, p. 141-163, 2007.

TORNESSEN, T. Process improvement and the human factor. Total Quality Management, v.11, n. 4/5, p. 773-778, 2005.

UPTON, D. M. Designing, Managing, and improving operations. New Jersey: Prentice Hall. 1998, 529 p. 